\title{
Vacuum (Meta)Stability Beyond the MSSM
}

\author{
Kfir Blum, Cédric Delaunay, Yonit Hochberg \\ Department of Particle Physics \\ Weizmann Institute of Science, \\ Rehovot 76100, Israel \\ kfir.blum@weizmann.ac.il, cedric.delaunay@weizmann.ac.il, \\ yonit.hochberg@weizmann.ac.il
}

\begin{abstract}
We study the stability of the Higgs potential in the framework of the effective Lagrangian beyond the MSSM. While the leading nonrenormalizable operators can shift the Higgs boson mass above the experimental bound, they also tend to render the scalar potential unbounded from below. The destabilization is correlated with the Higgs mass increase, so that if quantum corrections are small the problem is severe. We show that a supersymmetric sub-leading correction stabilizes the potential within the domain of validity of the effective theory. Constraints on MSSM parameters as well as on higher dimensional operators are derived, ensuring that our vacuum has a lifetime longer than the present age of the universe. In addition we show that when effective operators are responsible for evading the LEP bound, stability constraints imply an upper bound on the scale of new physics in the few $\mathrm{TeV}$ range.
\end{abstract}




\section{Introduction}

The Higgs tree level quartic couplings in the Minimal Supersymmetric Standard Model (MSSM) are completely dictated by gauge interactions. This fact stands at the heart of theoretical and phenomenological difficulties, perhaps foremost among them the tree level prediction that the lightest Higgs boson be lighter than the $Z$, a prediction ruled out by collider experiments [1, 2]. In order to evade the experimental bound on the Higgs mass large quantum corrections are required in the MSSM, implying a substantial hierarchy between the electroweak (EW) scale and the scale of supersymmetry (SUSY) breaking. In particular, at least one of the top superpartners is required to be much heavier than the top such that some amount of fine-tuning is needed [3, 4, 5, 6]. The fine-tuning becomes even more pronounced if the model is expected to provide explanations for present cosmological data. While the MSSM possesses all of the ingredients needed in order to account for both the dark matter and the baryon asymmetry of the universe, analyses reveal that difficulties associated with the Higgs mass bound are rooted in the cosmological arcade, too (see, for example [7, 8, 9]).

The restricted structure of the Higgs sector also makes it susceptible to small corrections from new physics Beyond the MSSM (BMSSM). If the scale associated with the BMSSM physics lies well above MSSM particle masses, an effective field theory approach becomes useful. The effective theory framework of the BMSSM was previously studied, e.g., in [10, 11, 12. The authors of [11] showed that the effective expansion takes on a rather simple form. In fact, under mild assumptions the leading nonrenormalizable corrections to the Higgs sector are captured by only two operators, one supersymmetric and the other associated with hard SUSY breaking (see also [13]). It was further demonstrated that these operators may lead to a sizable shift of the Higgs mass at the classical level. Besides potentially solving the difficulties mentioned above [14, 15, 16, this result is of considerable experimental importance as it opens up a zone of SUSY phenomenology in which both stops may be very light, possibly just around the corner for collider experiments.

However, examining the vacuum structure of the effective theory reveals that when the leading nonrenormalizable operators are taken to account for a significant shift for the lightest Higgs mass, they also destabilize the quartic couplings. Naively, this might be considered a severe setback to the picture drawn above. In the presence of a negative quartic coupling, stability relies on the higher order terms of the theory, which could a priori complicate the analysis considerably as well as introduce UV sensitivity. Nevertheless, we find that the effective theory exhibits a remarkable property: a single higher order operator arising at dimension six automatically cures the runaway initiated by the leading dimension five terms. Under mild assumptions, this operator is the only one relevant to the question of stability, and, furthermore, is directly correlated with the dimension five set. We find that the entire study of potential stability can be conducted and resolved within the range of validity of the effective approach. As a result, vacuum stability can be ensured by imposing simple relations between MSSM parameters and dimension five BMSSM operators, without the need to explicitly invoke the potentially far more complicated dimension six structure. In addition, we show that an upper bound on the scale of new physics may arise in the BMSSM, as well as derive the viable range for the lightest Higgs mass.

The interplay between the negative quartic and the higher dimensional operator often leads to the formation of a remote vacuum, in the presence of which the EW vacuum is only 
metastable. The existence of the remote vacuum was noticed by the authors of [17], where it was suggested that EW symmetry breaking may in fact lead our universe to reside in this new configuration dominated by nonrenormalizable operators. An intriguing feature of this scenario is that in this case, EW breaking may occur even in the SUSY limit. However, the structure of the effective theory implies that the EW scale be given in this case by the geometric mean of the new physics scale $M$ and the SUSY $\mu$ parameter $\sqrt{1}, v \sim \sqrt{\mu M}$. Null searches for charginos, for instance, highly restrict this possibility. In this paper we adopt a more conservative approach. We discuss the possibility that while the leading effective operators act to destabilize the potential, the usual EW breaking vacuum is either stable or of a life time longer than the present age of the universe.

The outline of this paper is as follows. In Section 2 we review the BMSSM Higgs sector, emphasizing the correlation between the lightest Higgs mass and the appearance of directions in field space where the potential is unbounded from below at leading order. We then study the next set of higher dimensional operators, showing that they can stabilize the potential up to the cutoff scale of the effective theory. In Section 3 we present analytical and numerical prescriptions ensuring the (meta)stability of the EW vacuum. Section 4 discusses the phenomenological implications of the stability constraints. Our conclusions are gathered in Section 5. Appendix $\mathrm{A}$ addresses the issue of charge breaking and $\mathrm{CP}$ violating field configurations, Appendix $\mathrm{B}$ elaborates on the quantum tunneling computation, and Appendix C presents constraints on the heavy cutoff scale arising from electroweak precision measurements.

\section{Higgs sector in the MSSM and Beyond}

In the bulk of this paper we analyze the vacuum stability of the BMSSM theory, namely the MSSM Higgs sector augmented by nonrenormalizable operators. It is useful to adopt the nomenclature of [11] and to classify the nonrenormalizable operators according to their scaling dimension, which is the total mass dimension of the fields contained in an operator, and their effective dimension, counting the powers of $1 / M$ which suppress it. For instance, an operator such as $(\mu / M) h_{u} h_{d}\left|h_{u}\right|^{2}+$ h.c. is of scaling dimension four but effective dimension five.

In Section 2.1 we briefly discuss the renormalizable MSSM Higgs sector, and highlight the main features which guide us in the study of nonrenormalizable corrections to it. In Section 2.2 we review BMSSM operators of effective dimension five. These operators alone suffice to lift the lightest Higgs mass above the LEP bound. However, as we show, these operators can destabilize the scalar potential at large field values which are still within the domain of validity of the effective theory. In that case, naively, the effective theory truncated at this order is not consistent; higher order operators must cure the instability. The next set of operators consists of effective dimension six, and we study it in Section 2.3 .

\footnotetext{
${ }^{1}$ The emergence of an intermediate scale of similar formal form was previously considered in [18, where it was discussed in the context of much higher energy phenomena.
} 


\subsection{MSSM setup}

The scalar Higgs potential of the renormalizable MSSM can be written as follow:2:

$$
\begin{aligned}
V_{\mathrm{MSSM}}= & m_{1}^{2}\left|h_{d}\right|^{2}+m_{2}^{2}\left|h_{u}\right|^{2}+\left(m_{12}^{2} h_{u} h_{d}+\text { h.c. }\right) \\
& +\frac{g_{Z}^{2}}{8}\left(\left|h_{u}\right|^{2}-\left|h_{d}\right|^{2}\right)^{2}+\frac{g^{2}}{2}\left(\left|h_{u}\right|^{2}\left|h_{d}\right|^{2}-\left|h_{u} h_{d}\right|^{2}\right),
\end{aligned}
$$

where $m_{1}^{2} \equiv m_{H_{d}}^{2}+|\mu|^{2}, m_{2}^{2} \equiv m_{H_{u}}^{2}+|\mu|^{2}, m_{12}^{2} \equiv B \mu$ and $g_{Z}^{2}=g^{2}+g^{\prime 2}$ with $g, g^{\prime}$ the SM gauge couplings. One can always choose a basis for the fields such that $m_{12}^{2}$ is real and positive, and we shall keep to such a basis consistently throughout the paper. We parameterize the expectation values as follows:

$$
\left\langle h_{u}\right\rangle^{T}=\left(0, \phi_{2}\right), \quad\left\langle h_{d}\right\rangle^{T}=\left(\phi_{1}+i \chi, \rho\right) .
$$

Gauge freedom is used to render $\left\langle h_{u}^{+}\right\rangle=0$ and $\phi_{2}$ real and positive, while the real component $\phi_{1}$ is allowed to obtain negative values 3 In the BMSSM, as we shall see, more than one vacuum configuration may develop. We will analyze paths of minimum potential energy which connect these vacua. Along such paths, in principle, it may become energetically favorable for non-vanishing values of $\chi$ or $\rho$ to turn on. This may occur even when all Lagrangian parameters are real, as we shall assume in this paper. In this case, we have found that ignoring the $\mathrm{CP}$ violating $(\mathrm{CPV})$ and charge-breaking $(\mathrm{CB})$ background fields $\chi$ and $\rho$ is typically well justified in the study of potential stability. Therefore, for clarity, we set $\rho=\chi=0$ in most of the paper. In places where deviations from this assumption become relevant we refer the reader to the discussion in Appendix A.

In the framework we study, $\mathrm{SU}(2)_{\mathrm{L}} \times \mathrm{U}(1)_{\mathrm{Y}}$ breaking into $\mathrm{U}(1)_{\mathrm{EM}}$ occurs as usual by an interplay between the quadratic and quartic terms in the scalar potential. (For an alternative scenario, see [17].) The resulting vacuum is parameterized by

$$
\begin{gathered}
v^{2}=\phi_{1}^{2}+\phi_{2}^{2} \simeq(174 \mathrm{GeV})^{2}, \\
\tan \beta=\phi_{2} / \phi_{1} .
\end{gathered}
$$

We call this vacuum the EW vacuum.

Being exclusively dictated by gauge superfield D-terms, the quartic couplings in (1) are proportional to the electroweak gauge couplings, making the Higgs sector sensitive to small corrections. In particular, quantum corrections arising from loops of MSSM fields with a large amount of SUSY breaking are usually conceived to account for a sizable shift in the lightest Higgs mass. In a similar manner, the quartic Higgs structure is also sensitive to nonrenormalizable operators, which need only compete with couplings of order $g^{2}$ in order to modify the spectrum.

Moreover, the quartic terms of (11) vanish along the D-flat directions, specified by $\left|\left\langle h_{u}\right\rangle\right|=$ $\left|\left\langle h_{d}\right\rangle\right|$. At tree (and renormalizable) level, this gives rise to a constraint on the quadratic

\footnotetext{
${ }^{2}$ We write the superfield components as $H=(h, \psi, F)$, the MSSM $\mu$ term as $\int d^{2} \theta \mu H_{u} H_{d}$, and our convention for $\mathrm{SU}(2)$ contraction is $H_{u} H_{d}=H_{u}^{+} H_{d}^{-}-H_{u}^{0} H_{d}^{0}$.

${ }^{3}$ Note that, in the renormalizable MSSM, the minimization conditions lead to $\rho=\chi=0$. In that case one may write the scalar potential using non-negative $\phi_{1}$ and $\phi_{2}$.
} 
terms, $m_{1}^{2}+m_{2}^{2}>2 m_{12}^{2}$, which must hold for the potential to be bounded from below. When nonrenormalizable operators are considered, the relative flatness of the renormalizable potential along the D-flat directions makes it important to verify that the higher dimensional operators do not destabilize the vacuum.

\subsection{Operators of effective dimension five}

Effective dimension five operators composed purely of Higgs fields enter the Lagrangian via the superpotential. Including F-term SUSY breaking, there are two such operators [11]:

$$
\frac{1}{M} \int d^{2} \theta\left(\lambda_{1}\left(H_{u} H_{d}\right)^{2}+\lambda_{2} \mathcal{Z}\left(H_{u} H_{d}\right)^{2}\right)+\text { h.c. }
$$

Here $\mathcal{Z} \equiv m_{S} \theta^{2}$ is a dimensionless chiral superfield spurion. One could also contemplate the existence of operators arising from D-term SUSY breaking, in which case additional effective dimension five operators arise. In this paper, however, we assume that the effect of D-term breaking is somehow suppressed or non-existent [11, 19].

We assume that the new physics generating the effective operators is approximately supersymmetric, $m_{S} \ll M$. We are interested in the imprint which the effective dimension five operators have on both the spectrum and the stability of the scalar potential, as we now discuss. At effective dimension five, the correction to the scalar potential resulting from (4) reads:

$$
\delta V_{5}=2 \epsilon_{1} h_{u} h_{d}\left(\left|h_{u}\right|^{2}+\left|h_{d}\right|^{2}\right)+\epsilon_{2}\left(h_{u} h_{d}\right)^{2}+\text { h.c. }
$$

where we have defined $\epsilon_{1} \equiv \lambda_{1} \mu^{*} / M$ and $\epsilon_{2} \equiv-\lambda_{2} m_{S} / M$. Expanding to order $\mathcal{O}(\epsilon)$, the following shift is obtained for the light (CP-even) Higgs boson mass:

$$
\delta_{\epsilon} m_{h}^{2}=2 v^{2}\left(\epsilon_{2 r}-2 \epsilon_{1 r} \sin 2 \beta-\frac{2 \epsilon_{1 r}\left(m_{A}^{2}+m_{Z}^{2}\right) \sin 2 \beta+\epsilon_{2 r}\left(m_{A}^{2}-m_{Z}^{2}\right) \cos ^{2} 2 \beta}{\sqrt{\left(m_{A}^{2}-m_{Z}^{2}\right)^{2}+4 m_{A}^{2} m_{Z}^{2} \sin ^{2} 2 \beta}}\right),
$$

where $\epsilon_{k r}$ denotes the real part of $\epsilon_{k}$. As explained in [11, only the real parts of $\epsilon_{1,2}$ enter the spectrum at leading order while the imaginary parts contribute to interactions and mixing. In this paper we make use of the fact that the spectrum is relatively insensitive to the imaginary parts of $\epsilon_{1,2}$ and consider, for simplicity, only the case where there exists a basis for $h_{u}, h_{d}$ in which $\epsilon_{1,2}$ are real and $m_{12}^{2}$ is real and positive. Henceforth we drop the $r$ subscript and assume that $\epsilon_{1,2}$ are real.

The contribution of the non-supersymmetric term to the mass shift is suppressed compared to the supersymmetric one. For example, in the limit $m_{A}^{2} \gg m_{Z}^{2}$ we have

$$
\frac{m_{h}^{2}}{m_{Z}^{2}}=\cos ^{2} 2 \beta+\frac{4 \epsilon_{2} \sin ^{2} 2 \beta}{g_{Z}^{2}}-\frac{16 \epsilon_{1} \sin 2 \beta}{g_{Z}^{2}}+\mathcal{O}\left(\frac{m_{Z}^{2}}{m_{A}^{2}}\right) .
$$

It follows that $\left|\delta_{\epsilon_{2}} m_{h}^{2} / \delta_{\epsilon_{1}} m_{h}^{2}\right| \approx\left|\epsilon_{2} / \epsilon_{1}\right| \sin 2 \beta / 4$, and so $\epsilon_{2}>4\left|\epsilon_{1}\right|$ is needed in order for both terms to give a comparable mass shift. In Figure 1 we report the size of both the SUSY preserving and breaking operators, as required for $m_{h}=115 \mathrm{GeV}$, illustrating that 

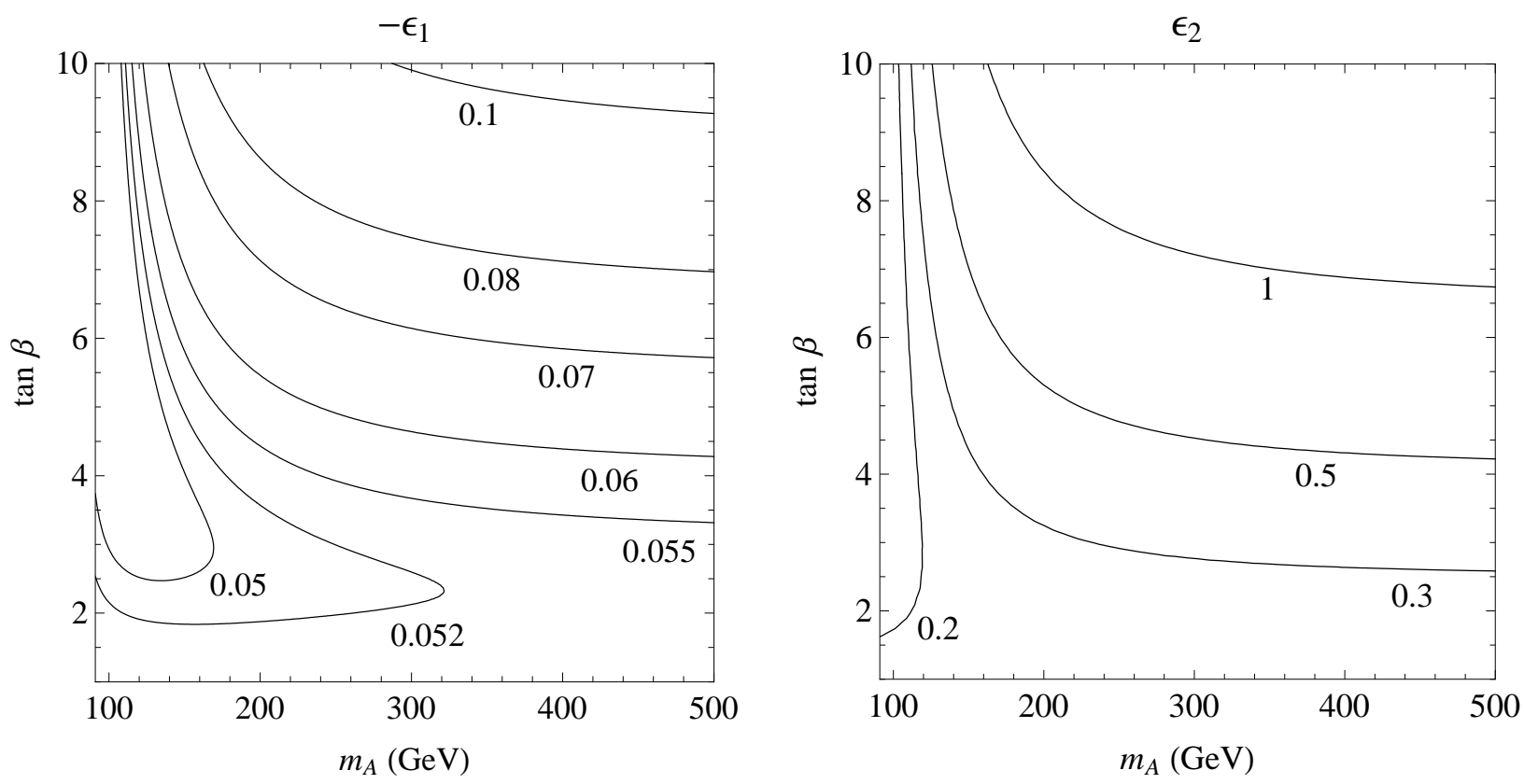

Figure 1: Contour plots of the values of $-\epsilon_{1}$ (left) and $\epsilon_{2}$ (right) corresponding to a fixed Higgs mass $m_{h}=115 \mathrm{GeV}$. In each panel only the considered operator is nonzero.

the former can easily lift the Higgs mass classically at this order, while existence of only the latter calls for large quantum corrections as in the MSSM. For moderate $\tan \beta$ a negative $\epsilon_{1}$ of magnitude $\left|\epsilon_{1}\right| \gtrsim 10^{-2} \times\left(1-r^{-2}\right) \tan \beta$, with $r \equiv m_{A} / m_{Z}$, can lift the Higgs mass above the LEP bound at tree level, without the need for quantum corrections. The large $\tan \beta$ limit is more involved. For $\tan \beta>\left|1 / \epsilon_{1}\right|$, the leading contribution of effective dimension five operators is suppressed to the level of the next order in $\epsilon$ and, as a result, becomes comparable to that of effective dimension six terms.

Apart from affecting the local properties of the vacuum (e.g. particle spectrum and interactions) the effective dimension five operators also influence the potential at large field values. Along the D-flat directions the potential is only bounded from below as long as $4\left|\epsilon_{1}\right|<\epsilon_{2}$. If this relation does not hold, the potential eventually becomes unstable and a runaway occurs along the direction $\phi_{1}=\operatorname{sign}\left(\epsilon_{1}\right) \phi_{2}$. In the interesting case where $\epsilon_{1}<0$, corresponding to a positive shift to the lightest Higgs mass, a saddle point emerges at field values

$$
-\phi_{1}=\phi_{2}=\sqrt{\frac{m_{1}^{2}+m_{2}^{2}+2 m_{12}^{2}}{8\left(\left|\epsilon_{1}\right|-\frac{1}{4} \epsilon_{2}\right)}},
$$

after which the potential decreases indefinitely. An important property of the runaway is that it occurs at field values $\phi^{2} \sim m^{2} / \epsilon \sim m M$, where $m$ refers to a combination of relevant quadratic mass parameters (presumably of electroweak scale) and $M$ is the heavy scale. Therefore, the runaway develops at $\phi \ll M$, rendering the EW vacuum unacceptably short-lived.

\footnotetext{
${ }^{4}$ Note that there are now two such directions as in our convention $\phi_{1}$ can be negative.
} 
Given the discussion above, one might naively conclude that the inequality $4\left|\epsilon_{1}\right|<\epsilon_{2}$ must be enforced as a physical constraint on the relative magnitudes of the supersymmetric vs. the SUSY breaking corrections [12]. In particular, in the supersymmetric limit wherein $\epsilon_{2}=0$, also $\epsilon_{1}=0$ would be required. However, as we show below, the supposed runaway is an artifact of the truncation of the effective expansion at $\mathcal{O}\left(M^{-1}\right)$ [20. This expansion cannot be trusted at large field values, even though it is consistent in the local vicinity of our vacuum [17]. Indeed, effective dimension six operators generate positive scaling dimension six contributions of the form $\phi^{6} / M^{2}$. Such terms eventually win over the negative quartic terms at field values $\phi^{2} \sim m M$, precisely the region of field space at which the instability starts to develop. Thus, effective dimension six operators are as important as the effective dimension five ones around the instability and may stabilize the potential before the UV threshold of the effective theory. Below we inspect the structure of effective dimension six operators in order to determine which ones are relevant to the stability problem. Compared to previous studies [21, 15], we find that focusing on the physical problem at hand simplifies the analysis considerably. Delightfully, we are able to show that over a broad range of parameters only one such operator exists, and even this operator not strictly independent from the dimension five set.

\subsection{Operators of effective dimension six}

We begin by considering effective dimension six operators which involve SUSY breaking. At order $1 / M^{2}$, there are no scaling dimension six operators which arise from SUSY breaking terms in the Lagrangian. The reason is that each term arising from SUSY breaking spurion must be accompanied by the appropriate power of the SUSY breaking mass scale. At scaling dimension six, this implies that the first (pure Higgs) operators are of order $1 / M^{3}$.

We do find effective operators associated with SUSY breaking spurions at scaling dimension four. These are of the form $(m / M)^{2} h^{4}$, where we extend the definition of $m$ to include the SUSY breaking scale, also of order the electroweak scale in this framework. Along the D-flat directions such contributions are suppressed by an additional power of $m / M$ in comparison to the effective dimension five terms of $\delta V_{5}$. Away from the D-flat directions, MSSM D-terms guarantee stability provided that we impose

$$
\epsilon^{2} \lesssim \frac{g_{Z}^{2}}{8} \approx \frac{1}{15}
$$

as a parametric inequality, where $\epsilon^{2} \equiv(\mathrm{m} / M)^{2}$ represents the magnitude of the dimensionless coefficient of such operators. Henceforth we restrict the discussion to the scenario in which Eq. (9) is satisfied. We shall see that this assumption simplifies the problem considerably. Moreover, it stands in accordance with requiring the effective theory expansion in powers of $\epsilon$ to remain valid, regardless of the stability analysis. Referring to Figure 1 we find that, at least for moderate $\tan \beta$, Eq. (9) does not pose any real limitation on the role of the $\epsilon_{1}$ correction in lifting the Higgs mass above the current experimental bound. Nevertheless, in general, the scaling dimension four, effective dimension six operators do not display any particular $\tan \beta$ dependence and so they can have a comparable influence on the spectrum in the large $\tan \beta$ regime, where the leading dimension five contributions are suppressed. Due 
to the multiplicity of independent coefficients, the analysis of the spectrum is involved in this case and we do not pursue it further in this paper.

From the discussion above we conclude that it is enough for our purpose to study effective dimension six operators in the supersymmetric limit. Squaring the $1 / M$ piece of the F-term equations of motion, arising in the presence of the supersymmetric effective dimension five operator gives

$$
\delta V_{6}=4\left|\frac{\epsilon_{1}}{\mu}\right|^{2}\left|h_{u} h_{d}\right|^{2}\left(\left|h_{u}\right|^{2}+\left|h_{d}\right|^{2}\right) .
$$

The contribution (10) is positive definite and non-vanishing along the D-flat directions. Thus it plays an important role in stabilizing the potential. Note that, for a given value of the $\mu$ parameter, the coefficient of this contribution is correlated with the Higgs mass through Eq. (6).

Superpotential operators which involve gauge superfields contribute to the scalar potential at effective dimension six, and must contain D-term components. Kähler operators which arise at order $1 / M^{2}$ can only affect the scalar potential through either F-terms or D-terms, by gauge invariance. Considering F-terms, we immediately see that these cannot contribute to effective dimension six, scaling dimension six operators, since they are linear in $h$ at leading order in $1 / M$. F-terms do contribute to effective dimension six, scaling dimension four operators. However, following Eq. (9) and the related discussion, such contributions are parameterically suppressed and we need not pursue them further. Considering D-terms, and ignoring sub-leading scaling dimension four operators, we find scaling dimension six operators which are of the general form

$$
\frac{\tilde{g}^{2}}{M^{2}} h^{4}\left(\left|h_{u}\right|^{2}-\left|h_{d}\right|^{2}\right)
$$

where $\tilde{g}^{2}$ stands for some bilinear combination of $g$ and $g^{\prime}$.

We learn that, apart from the operator (10), all other scaling dimension six contributions to the scalar potential are gauge coupling suppressed and - most importantly - vanish along the D-flat directions of the MSSM. Therefore at large field values (but still $\ll M$ ) and along the would-be runaway direction described in the previous section, the potential is in fact driven by the positive definite scaling dimension six $\delta V_{6}$ given in (10). Thus the same superpotential operator responsible for lifting the Higgs mass classically also ensures that the scalar potential does not exhibit a runaway. The phenomenology of this correlation we aim to address in the next section.

\section{Vacuum stability}

In what follows we analyze the stability of the scalar potential including the dimension six operator identified in the previous section, namely $V=V_{\text {MSSM }}+\delta V_{5}+\delta V_{6}$. Simple analytical and numerical criteria which ensure the stability of a given potential configuration are formulated. These criteria can be put in terms of relations between the electroweak scale parameters and effective dimension five operators. 


\subsection{Analytical approximation}

It is useful to first analyze the potential along the MSSM D-flat directions. While soft terms, quantum corrections and the presence of the EW vacuum all play a role in shifting the potential features somewhat away from $\left|\left\langle h_{u}\right\rangle\right|=\left|\left\langle h_{d}\right\rangle\right|$, all of the insights are contained and, further more, it turns out to be a reasonable approximation to study the profile of the potential at these well defined directions in field space. We begin by performing this analysis at tree level, assuming vanishing $\chi$ and $\rho$ values. Then, having obtained the principal results we extend the discussion to include all of the complications mentioned above.

At tree level, the effective potential along the MSSM D-flat directions take the form:

$$
V^{\mathrm{D}-\mathrm{flat}}(\phi)=\frac{1}{2}\left(m_{2}^{2}+m_{1}^{2} \mp 2 m_{12}^{2}\right) \phi^{2}+2\left(\frac{\epsilon_{2}}{4} \mp \epsilon_{1}\right) \phi^{4}+\left|\frac{\epsilon_{1}}{\mu}\right|^{2} \phi^{6} .
$$

with $\pm \phi_{1}=\phi_{2} \equiv \phi / \sqrt{2}$. If the quartic coupling in (12) is negative, the potential may develop another vacuum away from the electroweak scale. Thus a simple way to guarantee stability is to impose $\epsilon_{2}>4\left|\epsilon_{1}\right|$. However, from Figure 1 we learn that typical values for $\left|\epsilon_{1}\right|$, consistent with the bound on the lightest Higgs mass are in the range $\left|\epsilon_{1}\right| \sim 0.05-0.1$. The simple condition for an always-positive quartic is therefore in some tension with the need to assure that higher order terms are under control (see Eq. (9)). Hence we attend to the more interesting case where the quartic coupling is negative. It is useful to define the quantities $m^{2} \equiv m_{2}^{2}+m_{1}^{2} \mp 2 m_{12}^{2}$ and $\tilde{\epsilon} \equiv \epsilon_{2} / 4 \mp \epsilon_{1}$. Using these quantities we see that the non-zero extrema of the potential, corresponding to the remote vacuum and saddle point are located respectively at

$$
\phi^{2}=-\frac{2|\mu|^{2}}{3 \tilde{\epsilon}}\left(\frac{\tilde{\epsilon}}{\epsilon_{1}}\right)^{2}\left[1 \pm \sqrt{1-\frac{3 m^{2}}{8|\mu|^{2}}\left(\frac{\epsilon_{1}}{\tilde{\epsilon}}\right)^{2}}\right] .
$$

Manipulating Eqs. (12) and (13) we arrive at the following criterion, designed to ensure that the remote minimum along the D-flat direction is at most degenerate with, but never deeper than the potential at the origin of field space:

$$
\frac{m^{2}}{|\mu|^{2}} \geq 2\left(\frac{\tilde{\epsilon}}{\epsilon_{1}}\right)^{2}
$$

A remarkable feature of the condition (14) is that it does not involve the heavy scale $M$. Rather, it involves a relation between relevant mass parameters of the MSSM and the relative size of nonrenormalizable operators. Indeed, from Eq. (13) it follows that both the remote vacuum and the saddle point scale similarly, as $\phi \sim \sqrt{m M} \ll M$. This behavior reflects the fact that the quartic couplings along the D-flat directions originate from effective dimension five operators. As a result, there exists a scale at which quadratic, quartic and dimension six contributions in the potential are of similar magnitude; this scale is precisely $\sqrt{m M}$.

For concreteness we attend to the case $\epsilon_{1}<0$, corresponding to a positive shift for the lightest Higgs mass. Rewriting Eq. (14) in terms of physical quantities and to leading order 
in $\epsilon$, we arrive at the following useful relation:

$$
\frac{m_{A}^{2}(1+\sin 2 \beta)}{|\mu|^{2}} \geq 2\left(\frac{\tilde{\epsilon}}{\epsilon_{1}}\right)^{2}\left[1+\frac{m_{Z}^{2}}{m_{A}^{2}} \frac{16 \tilde{\epsilon}}{g_{Z}^{2}}\left(\frac{1+2 \sin 2 \beta}{1+\sin 2 \beta}-\frac{3}{2} \frac{\epsilon_{1}}{\tilde{\epsilon}}\right)\right]^{-1} .
$$

Restricting to the D-flat direction allowed us to write a stability criterion of simple analytical form. However, the actual vacua and saddle point emerge somewhat away from the D-flat direction. In particular, the remote vacuum (and, similarly, saddle point) can sustain an angle $\sim m / \sqrt{m M} \sim \sqrt{\epsilon}$ from the flat direction. Hence imposing stability along $\left|\phi_{1}\right|=\left|\phi_{2}\right|$ does not forbid the actual remote vacuum from becoming a global minimum. It is therefore important to complement Eq. (15) using numerical methods. In practice, as we show below, Eq. (15) turns out to be a robust but slightly conservative stability criterion.

\subsection{Numerical approach}

Armed with intuition from the analytical analysis, we proceed to define stability criteria based on numerical procedures. We formulate two such criteria, then comment on the possibility of CPV or CB field configurations.

Vacuum degeneracy First, we define a stability criterion by computing the potential and numerically verifying that the remote vacuum is at most degenerate with, but never deeper than the EW vacuum. We call this constraint vacuum degeneracy; it is robust but conservative.

Quantum tunneling Second, we define a stability criterion by numerically computing the tunneling rate from the EW to the remote vacuum. The tunneling rate is given by $\Gamma \propto \exp (-B)$, with $B$ the bounce action. A metastable configuration is viable if the universe remains in the EW vacuum for longer than its age. Quantitatively, this translates into $B \gtrsim 400$. We have used an approximate method to compute the bounce action, and errors of $\mathcal{O}(1)$ are expected. Hence we discuss configurations with bounce action of $B=400$ and $10^{3}$. A detailed computation of the tunneling rate can be found in Appendix B.

In the discussion above we have ignored the $\mathrm{CP}$ violating and charge breaking background fields, $\chi$ and $\rho$. However, these effects are accounted for when we numerically search for vacuum degeneracy and compute the bounce action. We find that neglecting the $\chi$ and $\rho$ background fields is almost always justified in the analysis of potential stability in our framework, and does not affect the reliability of Eq. (15). The reason is that the stability criterion itself is typically sufficient to ban non-vanishing $\chi$ and $\rho$, for field values $\phi<M$. More details can be found in Appendix A.

In Figure 2 (three panels on the left) the stability criteria are projected on various sections of the parameter space, illustrating the above analysis. We focus on the combined study of electroweak scale parameters vs. the heavy scale $M$. The following statements can be made.

The analytical stability criterion is slightly less conservative than, but closely follows the robust numerical criterion of vacuum degeneracy. Moreover, in the parameter space depicted in Figure 2 we find that the analytical criterion is typically more conservative than imposing 
$B \gtrsim 10^{3}$, and always ensures that the bounce action will be above $B \sim 400$. Thus we can safely say that the analytical criterion is robust, at least for moderate values of $\tan \beta \lesssim 10$ where small $\epsilon_{1} \lesssim 0.1$ is more than sufficient to lift the Higgs mass above the experimental bound. Since the gap between the various criteria is modest at all parameter values, it is evident that Eq. (15) provides a detailed qualitative, as well as quantitative understanding of the parameter space of vacuum stability.

Regarding the tunneling action, we find that it rises steeply above $B \sim 100$, rendering the $B=400$ and $10^{3}$ contours very close to each other. This occurs since in the relevant regions of parameter space the remote minimum is nearly degenerate with the $\mathrm{EW}$ vacuum, and so a small change of parameters towards vacuum degeneracy causes the action to diverge.

\section{Phenomenological implications}

Finally the stage is set to study the implications of stability constraints. We begin by discussing relations between electroweak scale parameters and the effective dimension five operators. We then study constraints on the heavy BMSSM scale $M$. Lastly we describe the viable shift to the lightest Higgs mass, and outline how the analysis is altered in the presence of radiative corrections.

\subsection{Constraints on BMSSM parameters}

The parameter space consistent with vacuum stability, depicted in Figure 2 for $m_{h}=115$ $\mathrm{GeV}$, becomes smaller when the value of $m_{h}$ is increased further above the experimental bound 5 Hence Figure 2 can be interpreted as a translation of our current knowledge, $m_{h}>$ $114 \mathrm{GeV}$, into bounds on the viable ranges of other BMSSM parameters, subject to the assumption that quantum corrections are small. Furthermore, one of the merits of the analytical criterion Eq. (15) is that it allows to extract limiting relations between BMSSM parameters regardless of the precise value of the Higgs mass, as long as the expansion to order $\mathcal{O}(\epsilon)$ is valid in the local vicinity of the EW vacuum. In order to gain further insight it is useful to look at the behavior of Eq. (15) in the following two limits.

Large $m_{A}$ limit $-m_{A}^{2} \gg m_{Z}^{2}$ : In this case Eq. (15) implies

$$
|\mu| \lesssim m_{A}\left|\frac{\epsilon_{1}}{\tilde{\epsilon}}\right| \sqrt{\frac{1+\sin 2 \beta}{2}} .
$$

This condition is robust but conservative. In other words, while having $|\mu| \lesssim m_{A}\left|\epsilon_{1} / \tilde{\epsilon}\right|$ ensures stability, somewhat larger values of $|\mu| / m_{A}$ may still yield stable vacuum configurations, as illustrated in Figure 2. Indeed, comparing the two upper panels we see that while the vacuum degeneracy criterion requires $m_{A}>|\mu|$, the more loose tunneling action criterion allows $m_{A} \approx|\mu|$. For example, taking $\tan \beta=4, \epsilon_{2}=-\epsilon_{1}=0.06,|\mu|=m_{A}=3 m_{Z}$ gives

\footnotetext{
${ }^{5}$ A quick way to understand this statement (which will be assessed in Section 4.3) is by noting that, for given values of $m_{A}, \tan \beta$ and $\epsilon_{2}$, the way to increase $m_{h}$ is via making $\epsilon_{1}$ more negative. Vacuum instability is then driven stronger by the negative quartic $\sim \epsilon_{1} \phi^{4}$, balanced at large fields by the dimension six term $\sim \phi^{6} / M^{2}$. It follows that the upper bound on $M$ derived from stability must decrease as $m_{h}$ is increased.
} 

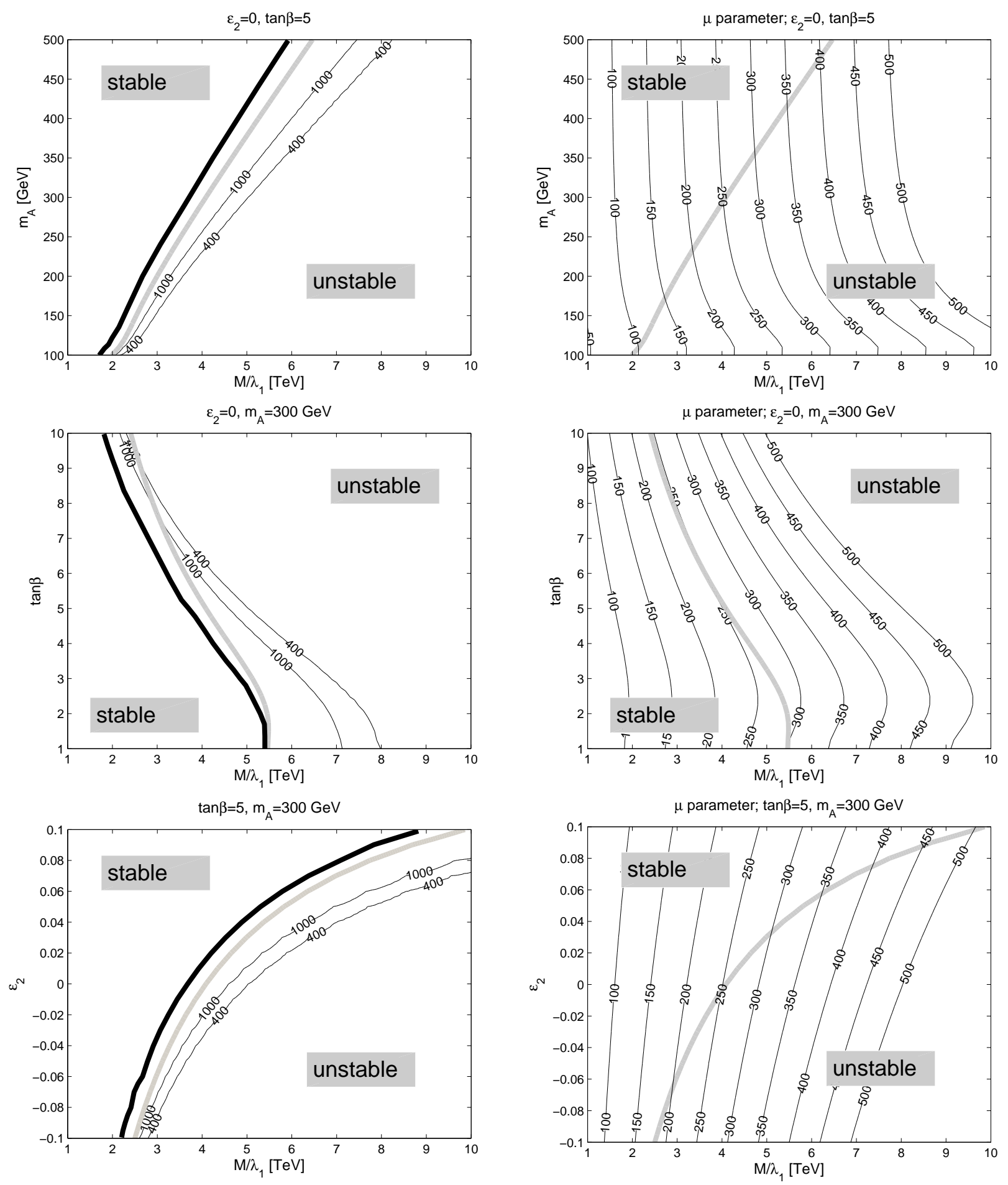

Figure 2: Regions of vacuum stability, projected on the heavy scale $M$ vs. electroweak scale parameters. All plots are generated at tree level, for fixed value of $m_{h}=115 \mathrm{GeV}$. Left panels: Stability criteria of Eq. (15), vacuum degeneracy and quantum tunneling are denoted by thick gray, thick black and thin labeled lines, respectively. To the left of each line the EW vacuum is stable according to that criterion, while to the right it is unstable. Right panels: Contours of $|\mu|$ (in $\mathrm{GeV}$ ) for the same parameter space of the left panels. The stability criterion of Eq. (15) is superimposed to enable easy comparison between the two columns. 
a stable vacuum configuration with $m_{h} \approx 120 \mathrm{GeV}$ at tree level. Note that, in order to guarantee vacuum stability, it is not a very good practice to assume very heavy $m_{A}$. The reason is that in order for the effective theory to work properly, it is necessary to retain some hierarchy between the high scale $M$ and electroweak mass parameters, and so taking any of the latter very large can pose a problem. However, positive values for the SUSY breaking $\epsilon_{2}$ result in a cancellation in $\tilde{\epsilon}$ and so the stability criteria can easily be satisfied through a combination of moderate ratios $m_{A} /|\mu| \sim 1$ and $\left|\epsilon_{1} / \tilde{\epsilon}\right| \gtrsim 1$.

Small quartic coupling limit $-\left|\tilde{\epsilon} / \epsilon_{1}\right| \ll 1$ : An accidental cancellation between the SUSY preserving and breaking dimension five operators can lead to $\left|\tilde{\epsilon} / \epsilon_{1}\right| \ll 1$. In this case, for negative $\epsilon_{1}$, the RHS of Eq. (15) approaches zero from above as the ratio $\left|\tilde{\epsilon} / \epsilon_{1}\right|$ decreases, and the stability constraint becomes trivially satisfied. A vanishing $\tilde{\epsilon}$ corresponds to large $\epsilon_{2}>0.1$ if the LEP bound is satisfied via $\epsilon_{1}$. A finite ratio is then required for the effective expansion to remain valid. However, the role of the non-supersymmetric operator $\epsilon_{2}$ is clear. While it may not much affect the Higgs mass, this term may be important in stabilizing the potential; it thus partially decouples the stability problem from the spectrum. The importance of $\epsilon_{2}$ for vacuum stability is illustrated in the lower panels of Figure 2. We see that turning on even a small positive value for $\epsilon_{2}$ rapidly opens up the parameter space corresponding to a stable EW vacuum. Note also that the $\mu$ parameter is largely unaffected by a change of $\epsilon_{2}$ when the Higgs mass is held fixed. This reflects the fact that the stabilization of the potential occurs at large field values.

\subsection{Constraints on the scale of new physics}

We now proceed to discuss the constraints imposed by vacuum stability on the scale of new physics $M$. To this end, note that the $\mu$ parameter can be traded for $M$ using $\mu=\epsilon_{1}^{*} M / \lambda_{1}^{*}$. We find that imposing stability implies an upper bound on the heavy scale $M$. For example, assuming $m_{A} \gtrsim 3 m_{Z}$ we obtain from Eq. (15):

$$
\left|\frac{M}{\lambda_{1}}\right| \lesssim \frac{m_{A}}{|\tilde{\epsilon}|} \sqrt{\frac{1+\sin 2 \beta}{2}} .
$$

The upper bound on $M$ for various parameter settings can be read off of Figure 2. Increasing $m_{A}, \epsilon_{2}$ or lowering $\tan \beta$ all act to weaken the bound; this behavior is readily understood from Eq. (17). At $\tan \beta=5$ and with $|\tilde{\epsilon}|=0.05$, for instance, we obtain $\left|M / \lambda_{1}\right| \lesssim 17 m_{A}$.

Of course, while it is the main issue of the current paper, vacuum stability is not the sole source of constraints on the scale of new physics. As an example, in Appendix C we show that electroweak precision tests (EWPTs) result in a lower bound for the heavy scale $M$,

$$
M \gtrsim 8 \mathrm{TeV}
$$

Put in conjunction with the stability constraints, a lower bound like (18) points towards a large value for $m_{A}$, sizable SUSY breaking $\epsilon_{2}$, a small tan $\beta$ or some combination of the above. Since the dimension six operators responsible for the leading Higgs mass shift and for potential stabilization are different from the ones which affect EWPTs, the combined 
bounds on $M$ may be interpreted in two ways. On the one hand, one may assume generic structure for the dimensionless coefficients of all nonrenormalizable BMSSM operators. In this case, if quantum corrections are small (e.g., say, both stops are found at the LHC), then taking the bound (18) together with the stability constraints leaves a very narrow range for the scale of the heavy BMSSM physics. Conversely, one may interpret these two bounds as hinting towards some suppression pattern in the microscopic extension behind the BMSSM theory.

\subsection{The lightest Higgs mass}

The stability criteria can also be expressed in terms of constraints on the lightest Higgs mass. We choose to present the resulting relation between $m_{h}$ and the heavy scale $M$. For clarity, we consider $m_{A} \gtrsim 3 m_{Z}$ and expand to zeroth order in $m_{Z}^{2} / m_{A}^{2}$. Assuming a negative quartic $\tilde{\epsilon}<0$, we can convert Eq. (17) into a limit on $\epsilon_{1}$, put in terms of $m_{A}, \tan \beta, \epsilon_{2}$ and $M$. Plugging the stability constraint in this form into the expression for the Higgs mass, Eq. (7), we obtain

$$
\frac{m_{h}^{2}}{m_{Z}^{2}} \lesssim \cos ^{2} 2 \beta+\frac{4 \epsilon_{2} \sin 2 \beta(1+\sin 2 \beta)}{g_{Z}^{2}}+\frac{16 m_{A} \sin 2 \beta \sqrt{(1+\sin 2 \beta) / 2}}{g_{Z}^{2}\left|M / \lambda_{1}\right|} .
$$

It is illuminating to compare Eq. (19) with Eq. (7). In particular, notice that in the $\epsilon_{2}$ term a suppression factor of $\sin ^{2} 2 \beta$ appearing in the spectrum equation gets replaced by a larger factor of $\sin 2 \beta(1+\sin 2 \beta)$ in the stability constraint. This result is explained as follows. First, recall that vacuum stability is trivially ensured if the overall quartic coupling along the relevant $\mathrm{D}$-flat direction is non-negative, $\tilde{\epsilon} \equiv \epsilon_{2} / 4+\epsilon_{1} \geq 0$. Given some value of $\epsilon_{2}$, substituting $\epsilon_{1}=-\epsilon_{2} / 4$ (which saturates the relation with a vanishing $\tilde{\epsilon}$ ) into Eq. (7) gives the second term on the RHS of (19). Hence the last term on the RHS of (19) represents the extra gain due to allowing a negative $\tilde{\epsilon}$ in the detailed analysis. This gain can indeed be significant: for example, at $\tan \beta=5(3)$ the numerical coefficient in front of the ratio $m_{A} /\left|M / \lambda_{1}\right|$ equals $\sim 9(16)$, so that a sizable shift for the Higgs mass is possible even with $m_{A} \ll M$. In addition, if $\epsilon_{2} \approx 0$ (in the case where the Beyond-MSSM new physics threshold is supersymmetric, for instance) then the last term in Eq. (19) provides the maximal tree level mass shift consistent with vacuum stability.

The above analysis is illustrated in Figure 3. In the left panel we hold $m_{A}, \tan \beta$ and $\epsilon_{2}$ fixed. The thick curve depicts the full analytical constraint derived from Eqs. (7) and (17), for which Eq. (19) represents the large $m_{A}$ limit. (It is easy to verify that Eq. (19) follows this curve to a very good approximation.) In order to keep track of the value of $\mu$, and so of $\epsilon_{1}$, we plot contours of constant $|\mu|$ as thin labeled lines. In the right panel we explore the range of $m_{h}$ accessible in the BMSSM at tree level. We find that with large $m_{A}$, small $\tan \beta$ and a sizable $\epsilon_{2}$, values of $m_{h} \approx 140 \mathrm{GeV}$ can be reached at tree level, in a stable vacuum configuration, even for $\left|M / \lambda_{1}\right| \gtrsim 8 \mathrm{TeV}$. Considering the right panel of Figure 3 and using Eq. (7), we find that $\left|\epsilon_{1}\right|$ becomes larger than 0.1 at $m_{h} \gtrsim 148,130,113 \mathrm{GeV}$ for the black, the dark gray and the light gray curves, respectively. For larger values of $m_{h}$ the expansion to linear order in $\epsilon_{1}$ eventually begins to break down. Thus even if very low values of $M / \lambda_{1}$ are acceptable, the stability curve cannot be trusted to arbitrarily large $m_{h}$; we will touch upon this issue again in the next subsection. 

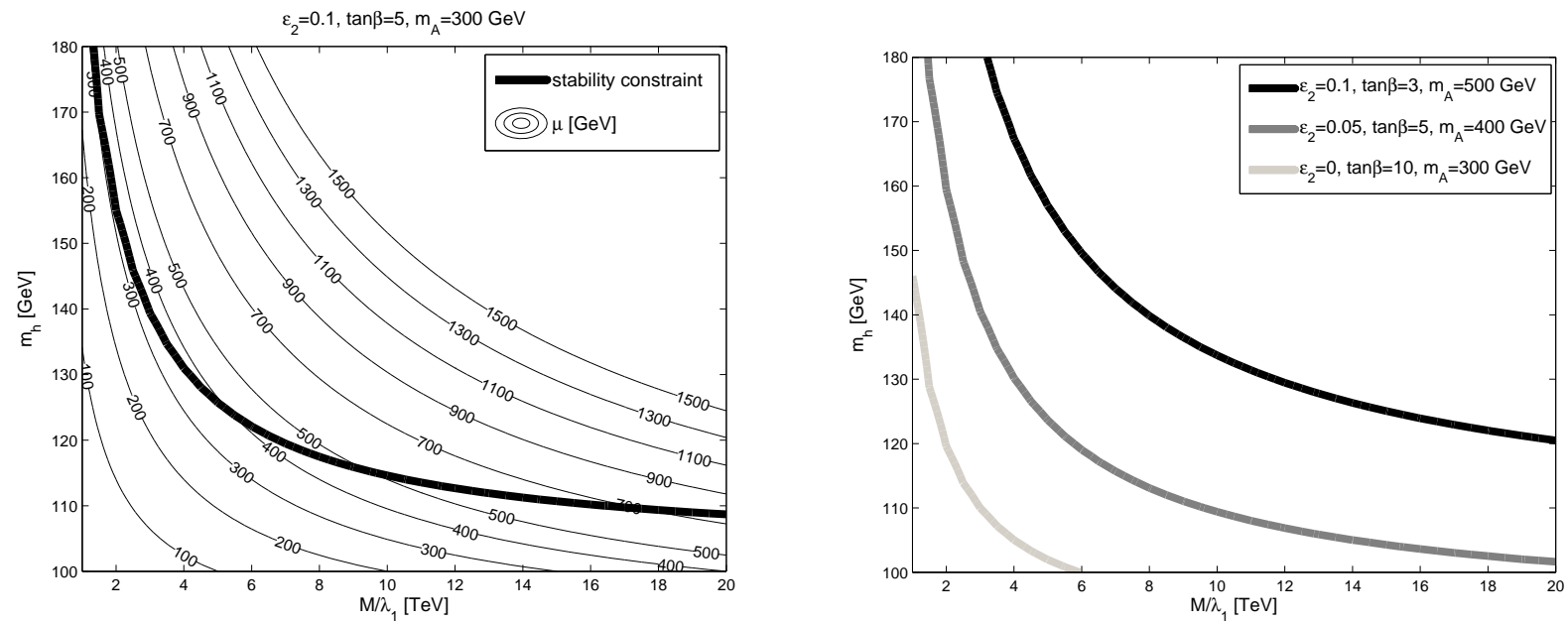

Figure 3: Left: Stability constraint in the $\left(m_{h}, M\right)$ plane, for $m_{A}, \tan \beta$ and $\epsilon_{2}$ as indicated. The thick line corresponds to the full analytical approximation (see text). Below this line the EW vacuum is stable. Thin contours denote values of $|\mu|$. Right: Stability constraint for different values of $\epsilon_{2}, m_{A}$ and $\tan \beta$.

\subsection{Quantum corrections}

The radiative corrections to the scalar potential are dominated by top and stop loops in the moderate $\tan \beta$ scenario. A (if not The) noteworthy feature of the BMSSM framework is that it allows quantum corrections to be small [11]. As already implied in this work, a completely supersymmetric top-stop sector is consistent with both the LEP bound on the lightest Higgs mass and stability considerations. Nevertheless, it is interesting to check to what extent our results are affected by moderate soft SUSY breaking masses for the stops. Here we do not attempt to study the stop sector in full detail. We find it sufficient for our purpose to neglect stop mixing and D-terms, adopt a common soft mass for both stops and work to one-loop order. Under these simplifications, quantum corrections modify the scalar potential by the expression:

$$
\delta V_{\tilde{t}} \approx \frac{3}{16 \pi^{2}}\left[m_{\tilde{t}}^{4}(\phi)\left(\ln \frac{m_{\tilde{t}}^{2}(\phi)}{Q^{2}}-\frac{3}{2}\right)-m_{t}^{4}(\phi)\left(\ln \frac{m_{t}^{2}(\phi)}{Q^{2}}-\frac{3}{2}\right)\right] .
$$

The field dependent masses are given by $m_{t}(\phi)=y_{t} \phi_{2}, m_{\tilde{t}}^{2}(\phi)=m_{t}^{2}(\phi)+m_{\text {stop }}^{2}$, with $m_{\text {stop }}$ the soft stop mass and $y_{t}$ the top Yukawa. $Q$ is the renormalization scale, which we choose to be $m_{Z}$. In writing Eq. (20), we impose renormalization conditions such that Eq. (3) remains unchanged. In Figure 4 we repeat the stability analysis with quantum corrections, focusing for concreteness on the $\left(M, m_{A}\right)$ and $\left(M, \epsilon_{2}\right)$ planes. We learn that soft masses for stops stabilize the potential, effectively removing the tree level upper bound on $M$ for $m_{\tilde{t}} \gtrsim 400$ $\mathrm{GeV}$.

Finally, Figure 5 depicts the effect of quantum corrections on the results derived for the lightest Higgs boson mass. Comparing with the right panel of Figure 3 we find, as expected, that quantum corrections increase the upper bound on $m_{h}$. A combination of small BMSSM operators $\epsilon \lesssim 0.05$ with modest radiative corrections from stops at the $200-250 \mathrm{GeV}$ range 
can easily and naturally accommodate the experimental bound on $m_{h}$, even for $M \gtrsim 10$ $\mathrm{TeV}$. Figure 5 includes the tree level numerical stability criterion of the tunneling action, as well as the analytical criterion Eq. (19). Comparing the two we find that the latter provides a reasonable, though slightly conservative approximation to the numerical bound for values of $m_{h}$ smaller than $\sim 140 \mathrm{GeV}$. For $m_{h} \gtrsim 140 \mathrm{GeV},\left|\epsilon_{1}\right|$ becomes larger than $\sim 0.13$ and the expansion to $\mathcal{O}(\epsilon)$ begins to fail, demonstrating the regime of validity of our analytical arguments.
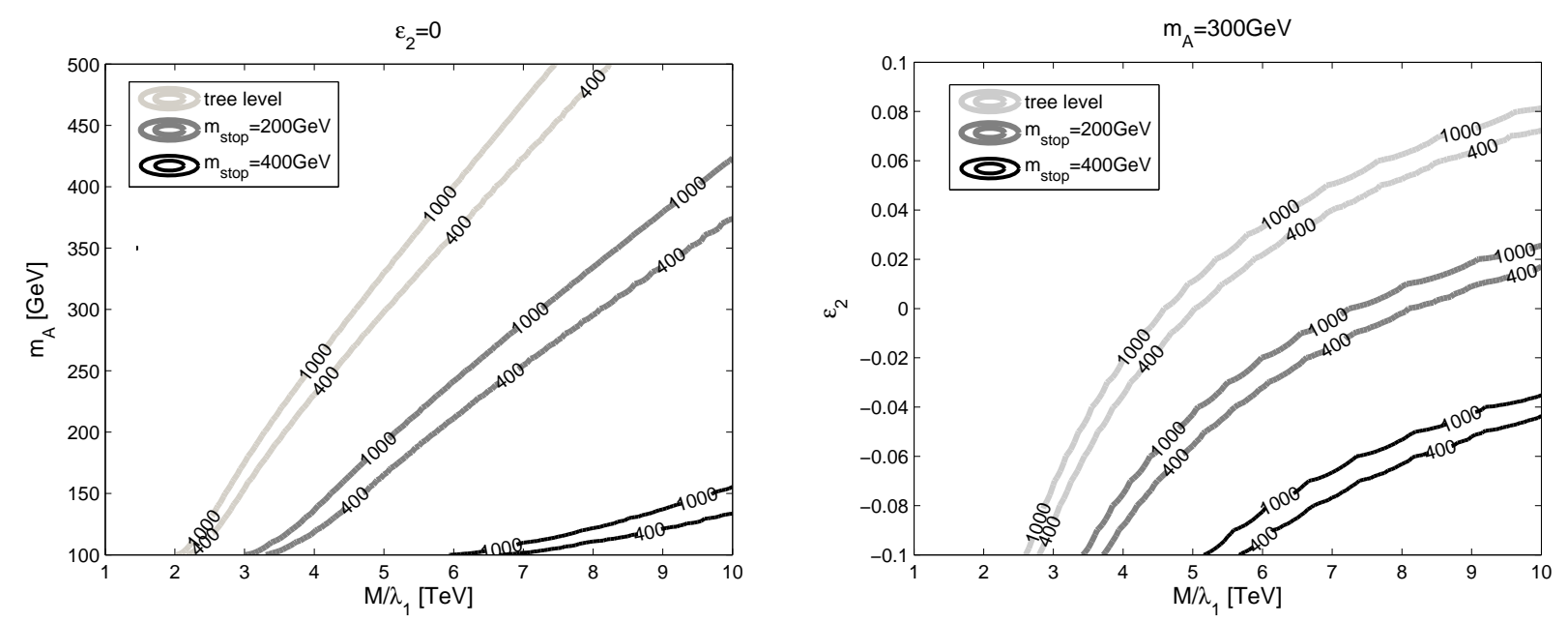

Figure 4: Contours of the tunneling action, including quantum corrections due to top and stop loops with a common soft stop mass of 0,200 and $400 \mathrm{GeV}$. The allowed parameter region lies to the left of the contours. The plots are generated for $\tan \beta=5$ and $m_{h}=115$ $\mathrm{GeV}$.

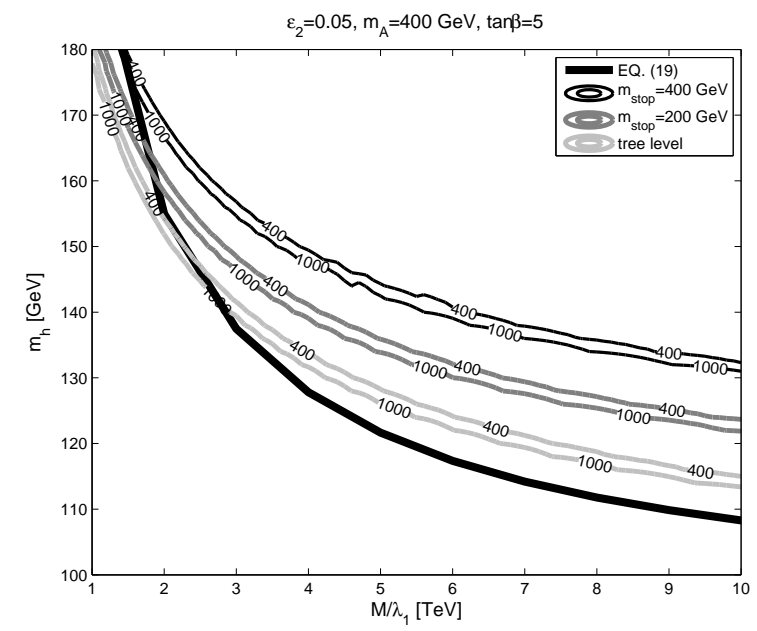

Figure 5: Contours of the tunneling action, including quantum corrections due to top and stop loops with a common soft stop mass of 0,200 and $400 \mathrm{GeV}$. The allowed parameter region lies below the contours. The plot is generated for fixed $m_{A}, \tan \beta$ and $\epsilon_{2}$ as indicated. 


\section{Conclusions}

In this paper we analyzed the vacuum structure of the BMSSM Higgs sector. We showed that the effective dimension five operators which lift the lightest Higgs mass are potentially harmful, as they are capable of destabilizing the scalar potential. It is easy to ensure that MSSM D-terms prevent the instability from occurring over most of the field space, for scales $\phi<M$, by imposing $\epsilon^{2} \lesssim 1 / 15$. This condition does not exclude a significant shift to the Higgs mass. Furthermore, it stands in accordance with the desire to keep the effective theory expansion under control. Along the D-flat directions, however, the MSSM D-terms vanish and the leading BMSSM correction is dominant. Thus if the quartic coupling along one of these directions is negative the effective expansion must be taken beyond leading order. Scrutinizing the effective theory to order $1 / M^{2}$, we were able to show that the stability of the scalar potential is controlled by only one scaling dimension six operator, which is supersymmetric and positive definite. This operator is correlated with the supersymmetric effective dimension five term which is the most relevant for lifting the lightest Higgs mass. Hence the nonrenormalizable part of the theory can cure itself, even though a remote vacuum may emerge before the cutoff scale of the effective theory.

In order to deal with a non-trivial potential structure, a set of criteria was developed from which relations between BMSSM parameters were inferred guaranteeing vacuum stability. In particular, by analyzing the potential along the D-flat directions we derived an approximate analytical criterion, Eq. (15), whose robustness was demonstrated by means of a full numerical study. Using this criterion we showed, for example, that if the LEP bound on the Higgs mass is accommodated at tree level in the BMSSM, then the stability of the EW vacuum is ensured provided that $m_{A} \gtrsim|\mu|$. Additionally, very low values of $\tan \beta \sim \mathcal{O}(1)$ are allowed and even favored despite the diminished MSSM contribution to the Higgs mass. Interestingly, at the classical level, vacuum stability implies an upper bound on the heavy scale $M$. This bound is better defined than what abstract fine-tuning arguments would suggest, and, since the experimental data can be accommodated with small $\mu / M \lesssim 0.05$, is also typically stronger. Put in conjunction with generic lower bounds on $M$, arising for instance from electroweak precision tests, the analysis either highly constrains the BMSSM parameter space or directs us towards a non-generic coupling structure in the effective theory.

Vacuum stability also dictates an upper bound on the lightest Higgs mass accessible via the leading effective operators. However, for $M$ in the few $\mathrm{TeV}$ range, $m_{h} \gtrsim 140 \mathrm{GeV}$ can still be accommodated at tree level, as a result of the potential stabilization provided by the supersymmetric dimension six operator. While a completely supersymmetric top-stop sector is allowed in the BMSSM (as far as the experimental bound on the lightest Higgs mass is regarded), quantum corrections due to stop loops are effective in stabilizing the potential, and two stop states at $\sim 300 \mathrm{GeV}$ suffice to significantly weaken the constraints due to vacuum stability. Indeed, light stops just around the corner for upcoming colliders can provide a relatively unimportant direct contribution for the Higgs spectrum, yet be significant for vacuum stabilization at large field values.

Finding both stops not too far above $m_{t}$ would already be a smoking gun for a BeyondMSSM extension. Information on the spectrum of charginos, neutralinos and the Higgs scalars, together with stability considerations, could then be used in order to extract additional constraints on dimension five operators and low energy MSSM parameters, as well as 
put an upper bound on the heavy scale $M$.

\section{Acknowledgments}

We thank Yossi Nir for fruitful discussions and Michael Dine, Zohar Komargodski and Nathan Seiberg for useful comments on the manuscript.

\section{A CP violating and charge breaking background fields}

Under the assumption of real parameters, the full effective potential including $\chi$ and $\rho$ fields is given by

$$
\begin{aligned}
V\left(\phi_{1}, \phi_{2}, \chi, \rho\right)= & m_{1}^{2}\left(\phi_{1}^{2}+\chi^{2}+\rho^{2}\right)+m_{2}^{2} \phi_{2}^{2}-2\left[m_{12}^{2}+2 \epsilon_{1}\left(\phi_{1}^{2}+\chi^{2}+\rho^{2}+\phi_{2}^{2}\right)\right] \phi_{1} \phi_{2} \\
& +2 \epsilon_{2}\left(\phi_{1}^{2}-\chi^{2}\right) \phi_{2}^{2}+\frac{g_{Z}^{2}}{8}\left(\phi_{1}^{2}+\chi^{2}+\rho^{2}-\phi_{2}^{2}\right)^{2}+\frac{g^{2}}{2} \phi_{2}^{2} \rho^{2} \\
& +4\left|\frac{\epsilon_{1}}{\mu}\right|^{2} \phi_{2}^{2}\left(\phi_{1}^{2}+\chi^{2}+\rho^{2}+\phi_{2}^{2}\right)\left(\phi_{1}^{2}+\chi^{2}\right) .
\end{aligned}
$$

First derivatives of interest follow,

$$
\begin{gathered}
\frac{\partial V}{\partial \chi^{2}}=m_{1}^{2}-4 \epsilon_{1} \phi_{1} \phi_{2}-2 \epsilon_{2} \phi_{2}^{2}+\frac{g_{Z}^{2}}{4}\left(\phi_{1}^{2}+\chi^{2}+\rho^{2}-\phi_{2}^{2}\right) \\
+4\left|\frac{\epsilon_{1}}{\mu}\right|^{2} \phi_{2}^{2}\left(2 \phi_{1}^{2}+2 \chi^{2}+\phi_{2}^{2}+\rho^{2}\right) \\
\frac{\partial V}{\partial \rho^{2}}=m_{1}^{2}-4 \epsilon_{1} \phi_{1} \phi_{2}+\frac{g_{Z}^{2}}{4}\left(\phi_{1}^{2}+\chi^{2}+\rho^{2}-\phi_{2}^{2}\right)+4\left|\frac{\epsilon_{1}}{\mu}\right|^{2} \phi_{2}^{2}\left(\phi_{1}^{2}+\chi^{2}\right)+\frac{g^{2}}{2} \phi_{2}^{2} .
\end{gathered}
$$

Since only the squares of $\chi$ and $\rho$ appear in the potential, a trivial extremum solution with $\chi=\rho=0$ always exists. CP violating (CPV) and charge breaking (CB) extrema require nontrivial solutions for a vanishing RHS in Eqs. (22) and (23), respectively.

We first derive the condition ensuring that the $\mathrm{EW}$ vacuum is $\mathrm{CP}$ and charge conserving. Demanding that the potential has a minimum at $\phi_{1}=v \cos \beta, \phi_{2}=v \sin \beta$ and $\rho=\chi=0$ and expanding to leading order in $\epsilon$, we obtain that an additional, nontrivial solution with $\phi_{1}=v \cos \beta, \phi_{2}=v \sin \beta$ and $\chi \neq 0$ is never allowed, while a solution with $\rho \neq 0$ requires $\rho^{2} \simeq-4 \sin ^{2} \beta\left(m_{A}^{2}+m_{W}^{2}+2 \epsilon_{2} v^{2}\right) / g_{Z}^{2}$. Hence $\chi=\rho=0$ is the only consistent solution at the EW vacuum, as long as:

$$
m_{A}^{2}+m_{W}^{2}+2 \epsilon_{2} v^{2}>0 .
$$

Considering Eq. (9) and the validity of the effective theory expansion, we find that this relation is always satisfied for $m_{A} \gtrsim m_{Z}$.

We now move on to find out how nonzero $\chi$ or/and $\rho$ affect the potential at large field values. In particular, we are interested in configurations of $\chi, \rho$ which extremize the potential 
in the vicinity of the tunneling path. Finding such extrema is important, since they may shift the saddle point and/or the remote vacuum from the $\left(\phi_{1}, \phi_{2}\right)$ plane and so alter the numerical computation of the stability criteria. Note that for large fields, unlike in the local neighborhood of the EW vacuum, one must keep the scaling dimension six operator appearing in the potential (21). In addition to the trivial solution $\{\chi=0, \rho=0\}$, there are three exclusive possibilities: $\{\chi \neq 0, \rho=0\},\{\chi=0, \rho \neq 0\}$ or $\{\chi \neq 0, \rho \neq 0\}$. To find out which of them is consistent, we rewrite the condition of vanishing right hand sides in Eqs. (22) and (23) as

$$
\chi^{2}=A_{C P V}\left(\phi_{1}, \phi_{2}\right)+B_{C P V}\left(\phi_{1}, \phi_{2}\right) \rho^{2}, \quad \rho^{2}=A_{C B}\left(\phi_{1}, \phi_{2}\right)+B_{C B}\left(\phi_{1}, \phi_{2}\right) \chi^{2} .
$$

Solutions with $\{\chi \neq 0, \rho=0\}$ or $\{\chi=0, \rho \neq 0\}$ only exist for $A_{C P V}>0$ or $A_{C B}>0$ respectively. Moreover, since the second derivatives of the potential with respect to $\chi^{2}$ and $\rho^{2}$ are positive definite, whenever one of these solutions becomes available within some region of the $\left(\phi_{1}, \phi_{2}\right)$ plane it will always minimize the potential energy, rendering the trivial solution unfavorable. Hence if the tunneling path traverses these regions, CPV or CB will turn on. A solution with $\{\chi \neq 0, \rho \neq 0\}$ requires that both $\chi^{2}=\left(A_{C P V}-A_{C B} B_{C P V}\right) /\left(1-B_{C B} B_{C P V}\right)$ and $\rho^{2}=\left(A_{C B}-A_{C P V} B_{C B}\right) /\left(1-B_{C B} B_{C P V}\right)$ be positive. If such a solution exists, it may or may not become energetically favored over solutions with only $\chi$ or $\rho$ nonzero.

We now describe how the $\chi$ and $\rho$ fields are dealt with in the numerical procedures presented in Section 3.2. The criterion of vacuum degeneracy requires computing the value of the potential at the remote minimum. For the tunneling action, according to the approximation we adopt (see Appendix $(\mathrm{B})$ ), the location of and potential value at both the remote minimum and saddle point are needed. To find these extrema in the presence of possible CPV and CB configurations, we numerically evaluate the full potential over the $\left(\phi_{1}, \phi_{2}\right)$ plane where $\chi$ and $\rho$ are replaced by the four classes of solutions defined above. Then we retain all consistent solutions extremizing the potential along both the $\phi_{1}$ and $\phi_{2}$ directions. While the remote minimum is unique, as it has to minimize the potential along all directions, there may be several saddle points corresponding to the different classes of solutions for $\chi$ and $\rho$. We compute the tunneling action assuming the path passes through the saddle point of minimum potential energy.

After the dust settles it turns out that in typical cases in which the $\mathrm{EW}$ vacuum is found to be stable, no CPV or CB arise in regions of field space that are relevant for the analysis, or otherwise the effect is very small. Thus the discussion conducted in Section 3.1, where for clarity we have set $\chi=\rho=0$, holds true up to minor changes. In order to see how this result comes about, it is useful once again to consider the problem close to the D-flat directions. Since away from them positive D-terms raise the potential, the tunneling path is confined to remain near this region. Thus removing CPV and CB from the flat directions protects the saddle point and the remote vacuum from developing nonzero $\chi$ or $\rho$. We can derive simple analytical criteria to exclude the formation of CPV or CB extrema along the D-flat directions. To this end, let us consider $\epsilon_{1}<0$ in which case potential instability occurs for negative $\phi_{1}$. Setting $-\phi_{1}=\phi_{2} \equiv \phi / \sqrt{2}$ and $\tilde{\epsilon} \equiv \epsilon_{2} / 4+\epsilon_{1}<0$, the extremum equations reduce to:

$$
-\left(\frac{g_{Z}^{2}}{4}+4\left|\frac{\epsilon_{1}}{\mu}\right|^{2} \phi^{2}\right) \chi^{2}=m_{1}^{2}+4\left(\frac{3 \epsilon_{1}}{2}-\tilde{\epsilon}\right) \phi^{2}+3\left|\frac{\epsilon_{1}}{\mu}\right|^{2} \phi^{4}+\left(\frac{g_{Z}^{2}}{4}+2\left|\frac{\epsilon_{1}}{\mu}\right|^{2} \phi^{2}\right) \rho^{2},
$$




$$
-\frac{g_{Z}^{2}}{4} \rho^{2}=m_{1}^{2}+\frac{g^{2}}{4}\left(1+\frac{8 \epsilon_{1}}{g^{2}}\right) \phi^{2}+\left|\frac{\epsilon_{1}}{\mu}\right|^{2} \phi^{4}+\left(\frac{g_{Z}^{2}}{4}+2\left|\frac{\epsilon_{1}}{\mu}\right|^{2} \phi^{2}\right) \chi^{2} .
$$

We find that CPV and CB are banned along the D-flat directions provided the following conditions hold, respectively:

$$
\frac{m_{A}^{2}(1+\sin 2 \beta)}{|\mu|^{2}} \geq \frac{4}{3} f(\beta)\left(\frac{\tilde{\epsilon}}{\epsilon_{1}}\right)^{2}\left(1-\frac{3 \epsilon_{1}}{2 \tilde{\epsilon}}\right)^{2}, \quad \frac{m_{A}^{2}(1+\sin 2 \beta)}{|\mu|^{2}} \geq f(\beta)\left(\frac{g^{2}}{8 \epsilon_{1}}+1\right)^{2}
$$

up to $\mathcal{O}\left(m_{Z}^{2} / m_{A}^{2}\right)$ corrections, where $f(\beta) \equiv(1+\sin 2 \beta) / \sin ^{2} \beta$ satisfies $1<f(\beta)<4$ for $\tan \beta>1$. These two conditions can be compared to the approximate analytical criterion derived in Section 3.1 which ensures that the EW vacuum is stable, namely $m_{A}^{2}(1+$ $\sin 2 \beta) /|\mu|^{2} \geq 2\left(\tilde{\epsilon} / \epsilon_{1}\right)^{2}$. Regarding CB, we find that vacuum stability guarantees no CB turns on along the D-flat direction provided that $\left|\epsilon_{1}\right| \lesssim 0.2$. In view of Figure 1, as well as considering Eq. (9) and the validity of the effective theory expansion, it is clear that this relation does not constitute a real compromise for the size of $\epsilon_{1}$. The appearance of CPV is a less marginal effect. However, the form of the inequalities (28) and the stability constraint implies that the stable parameter space exhibits little CPV; numerically we find that in most stable scenarios CPV does not occur along the tunneling path, or else does not encompass the saddle point nor the remote minimum. We conclude that imposing vacuum stability typically renders CB and CPV configurations irrelevant in the calculation. Note that since the tunneling path does not pass strictly along, but merely in the vicinity of the D-flat direction, the above analysis provides an intuitive argument only. However, as previously mentioned, numerically scanning the relevant parameter space we find that in practice it is indeed safe to neglect $\chi$ and $\rho$ in the analysis of vacuum stability in the BMSSM with real Lagrangian parameters.

\section{B Quantum tunneling}

We are interested in analyzing the stability of configurations wherein the potential exhibits a remote vacuum, in the presence of which our EW vacuum may be metastable. At zero temperature, one should compute the rate of quantum tunneling from the EW to the remote vacuum. For completeness, let us briefly review the theoretical set up before going into the details of our implementation. In a semi-classical approach the tunneling rate (per unit time and volume) is given by the WKB approximation [22]:

$$
\Gamma \simeq A \exp (-B), \quad B=S_{E}\left[\phi_{b}\right]-S_{E}\left[\phi_{\text {false }}\right],
$$

where $S_{E}[\phi]$ is the Euclidean action and $A$ is the determinant of the Gaussian fluctuations around $\phi_{b}$, the so-called bounce solution of the equation of motion. The precise value of the prefactor $A$ plays a minor role in comparison to the exponential suppression factor. Hence we make the conservative assumption that the prefactor saturates the highest scale in the BMSSM framework: $A \simeq M^{4}$. The bounce solution is $O(4)$ symmetric [23], i.e. is only a function of $r=\sqrt{x^{2}+t_{E}^{2}}$. It solves the equation of motion

$$
\frac{d^{2} \phi_{b}}{d r^{2}}+\frac{3}{r} \frac{d \phi_{b}}{d r}-\nabla V\left(\phi_{b}\right)=0
$$


subject to the "bouncing" boundary conditions: $\phi_{b}^{\prime}(0)=0$ and $\phi_{b}(\infty)=\phi_{\text {false }}$.

In configuration space, quantum tunneling proceeds by nucleation, growth and percolation of true vacuum bubbles surrounded by a metastable environment. At a given time $t$, the portion $\mathcal{P}(t)$ of the volume of the universe filled by true vacuum bubbles is controlled by the tunneling rate and the expansion of the universe through the following relation [24]

$$
\mathcal{P}(t)=1-\exp \left[-\frac{4 \pi}{3} \int_{0}^{t} d t^{\prime} \Gamma\left(t^{\prime}\right) d_{H}^{3}\left(t^{\prime}\right)\right],
$$

where $d_{H}(t) \sim t$ is the horizon distance. For the case of quantum tunneling $(T=0)$, the rate $\Gamma$, given by (29), is time independent. Hence today, for $t_{0} \sim 10^{10} \mathrm{yr} \sim e^{100} v^{-1}$, the portion of the universe in the stable phase is given approximately by:

$$
\mathcal{P}\left(t_{0}\right) \sim 1-\exp \left[-\mathcal{O}(10) \exp \left(4 \log \frac{M}{v}-B\right)\left(v t_{0}\right)^{4}\right] .
$$

Therefore to ensure that the metastable vacuum has a life time longer than the present age of the universe, or equivalently that $\mathcal{P}\left(t_{0}\right) \simeq 0$, it is enough to impose the following constraint on the bounce action:

$$
B \gtrsim 400+4 \log \frac{M}{v}
$$

where the second term, coming from the exponential prefactor $A$, contributes at most $\mathcal{O}(10 \%)$ for $M$ in the $\mathrm{TeV}$ range.

In a one-dimensional field space (as in the Standard Model), the correct bounce solution is easily found by use of a simple "shooting" numerical method. However, in the case of multiple fields, the search of the bounce solution is a non-trivial numerical task. We make use of several simplifying assumptions, justified in the BMSSM setup under consideration.

The first simplification consists of projecting the action on a given path in field space, which is known to be close to the correct tunneling trajectory. This path is fully characterized by a single curvilinear coordinate $\phi_{s}$, i.e. $\phi=\phi\left(\phi_{s}\right)$. The initial task is then greatly simplified as the problem has been reduced to a one dimensional search for the bounce solution. However such a projection is in general hard to estimate. One exception is when the potential extrema are close to alignment in field space. In this case the tunneling trajectory may be well approximated by a straight line joining the true and false vacua [25]. Projecting on such a path allows one to easily compute the bounce action using a traditional shooting method. We stress that this approach is consistent as the approximate alignment that it requires typically arises in the BMSSM. Indeed, as is apparent in Figure 6, both the remote vacuum and the saddle point separating it from the EW vacuum form close to the D-flat direction.

Second, we adopt a triangular approximation for the potential barrier, allowing us to use the clear prescription given in [26]. Thus we reserve the more cumbersome shooting method only as a check on the triangle calculation, verifying that the two methods agree at the level of $\mathcal{O}(10 \%)$. In Figure 6 we demonstrate the use of the triangle approximation in computing the tunneling action. 

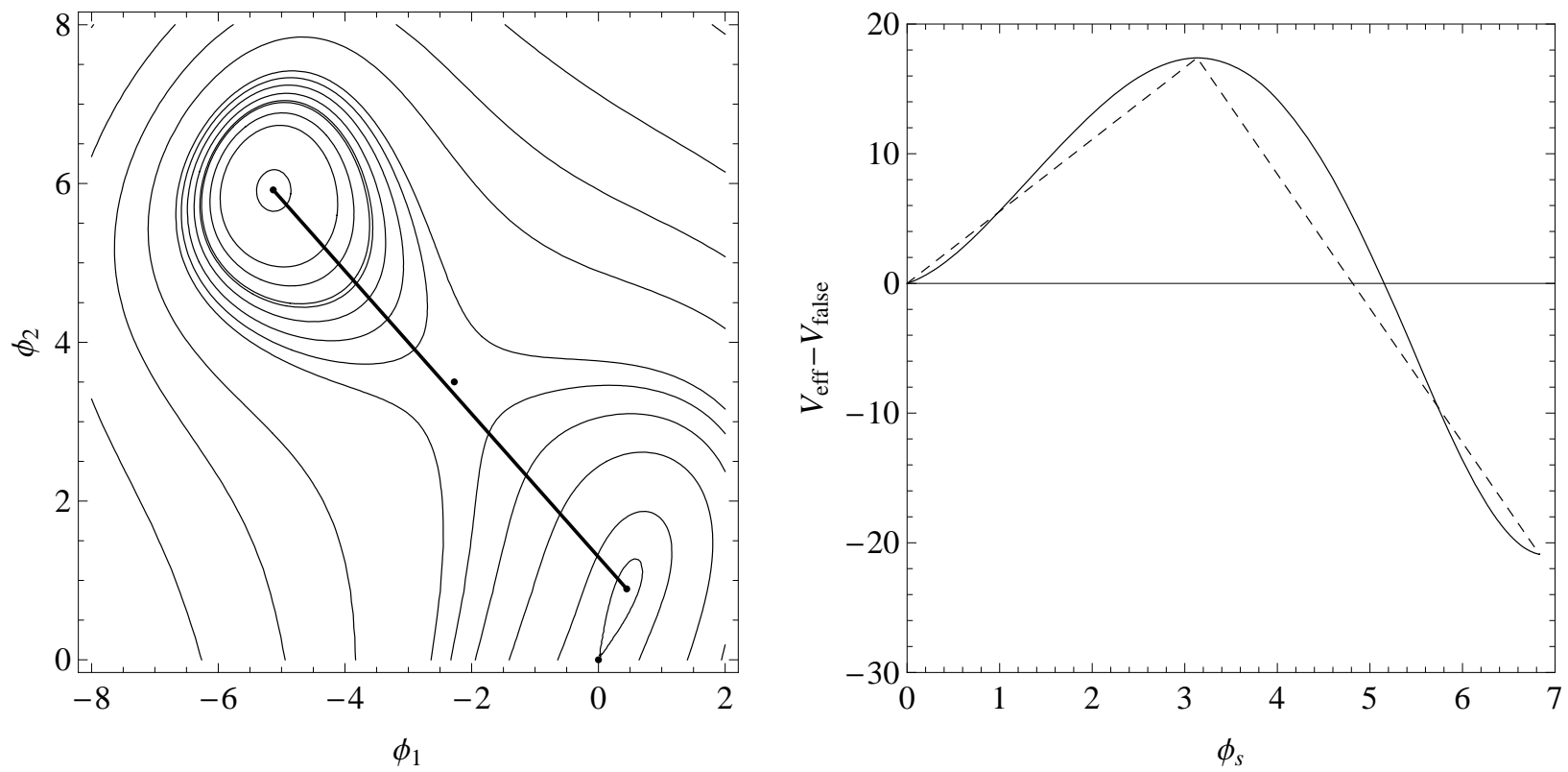

Figure 6: Left: Contour plot of the potential for $\mu=m_{A}=300 \mathrm{GeV}, \tan \beta=2, \epsilon_{2}=0$ and $\epsilon_{1} \simeq-0.05$, corresponding to tree level Higgs mass of $m_{h} \simeq 115 \mathrm{GeV}$. The straight line connects the two minima. Right: Potential projected on the straight line joining the two vacua (solid) and the corresponding triangle approximation (dashed) used to estimate the tunneling action. $\phi_{s}$ is the coordinate along the straight line. On both plots $\phi$ and $V_{\text {eff }}$ are in units of $v$ and $v^{4}$ respectively.

\section{Electroweak constraints}

As we have seen, stability of the scalar potential, arising from effective dimension six operators, gives an upper bound on the scale of new physics. Other effective dimension six operators involving the Higgs sector also affect the gauge terms in the Lagrangian, introducing mass shifts in the gauge sector as well as kinetic mixings. They are therefore constrained by electroweak data, in particular the precision electroweak variables $S$ and $T$ [27]

$$
S=-0.10 \pm 0.10, T=-0.08 \pm 0.11
$$

for $m_{h} \simeq 115 \mathrm{GeV}$. There are several effective dimension six operators involving the Higgs sector that can contribute to electroweak observables. For example, operators of the form $a_{i j}\left(H_{i}^{\dagger} e^{V} H_{i}\right)\left(H_{j}^{\dagger} e^{V} H_{j}\right) / M^{2}$ with $i, j=u, d$ in the Kähler potential contribute to the deviation of the $\rho$ parameter from unity by [10]

$$
\rho-1=-4\left(\frac{m_{W}}{g M}\right)^{2}\left[a_{u u} \sin ^{4} \beta+a_{d d} \cos ^{4} \beta-a_{u d} \cos ^{2} \beta \sin ^{2} \beta\right] \simeq \alpha T .
$$

For generic coefficients $a_{i j} \sim \mathcal{O}(1)$, the expression in square brackets in (35) is not particularly suppressed for any specific value of $\tan \beta$. We obtain a lower bound on the heavy scale,

$$
M \gtrsim 7.7 \mathrm{TeV} \text {. }
$$


Regarding the $S$ parameter, consider for instance a superpotential operator of the form $a_{W B} W^{\alpha} B_{\alpha} H_{u} H_{d} / M^{2}$, where $W_{\alpha}\left(B_{\alpha}\right)$ denotes the $S U(2)_{L}\left(U(1)_{Y}\right)$ gauge superfield strength. Such an operator generates a contribution to $S$ of

$$
S=\frac{32 \pi}{g g^{\prime}} \frac{v^{2} \sin 2 \beta}{2 M^{2}} a_{W B}
$$

Taking $a_{W B} \sim \mathcal{O}(1)$ we obtain a comparable bound, $M \gtrsim 8 \sqrt{\sin 2 \beta} \mathrm{TeV}$.

\section{References}

[1] R. Barate et al. [LEP Working Group for Higgs boson searches and ALEPH Collaboration and and], "Search for the standard model Higgs boson at LEP," Phys. Lett. B 565, 61 (2003) arXiv:hep-ex/0306033.

[2] S. Schael et al. [ALEPH Collaboration and DELPHI Collaboration and L3 Collaboration and ], "Search for neutral MSSM Higgs bosons at LEP," Eur. Phys. J. C 47, 547 (2006) arXiv:hep-ex/0602042.

[3] P. H. Chankowski, J. R. Ellis and S. Pokorski, "The fine-tuning price of LEP," Phys. Lett. B 423, 327 (1998) arXiv:hep-ph/9712234.

[4] R. Barbieri and A. Strumia, "About the fine-tuning price of LEP," Phys. Lett. B 433, 63 (1998) arXiv:hep-ph/9801353.

[5] P. H. Chankowski, J. R. Ellis, M. Olechowski and S. Pokorski, "Haggling over the fine-tuning price of LEP," Nucl. Phys. B 544, 39 (1999) arXiv:hep-ph/9808275.

[6] G. L. Kane and S. F. King, "Naturalness implications of LEP results," Phys. Lett. B 451, 113 (1999) arXiv:hep-ph/9810374.

[7] N. Arkani-Hamed, A. Delgado and G. F. Giudice, "The well-tempered neutralino," Nucl. Phys. B 741, 108 (2006) [arXiv:hep-ph/0601041].

[8] J. R. Ellis, S. F. King and J. P. Roberts, "The Fine-Tuning Price of Neutralino Dark Matter in Models with Non-Universal Higgs Masses," JHEP 0804, 099 (2008) arXiv:0711.2741 [hep-ph]].

[9] M. Carena, G. Nardini, M. Quiros and C. E. M. Wagner, "The Effective Theory of the Light Stop Scenario," JHEP 0810, 062 (2008) [arXiv:0806.4297 [hep-ph]].

[10] A. Brignole, J. A. Casas, J. R. Espinosa and I. Navarro, "Low-scale supersymmetry breaking: Effective description, electroweak breaking and phenomenology," Nucl. Phys. B 666, 105 (2003) arXiv:hep-ph/0301121.

[11] M. Dine, N. Seiberg and S. Thomas, "Higgs Physics as a Window Beyond the MSSM (BMSSM)," Phys. Rev. D 76, 095004 (2007) [arXiv:0707.0005 [hep-ph]]. 
[12] I. Antoniadis, E. Dudas, D. M. Ghilencea and P. Tziveloglou, "MSSM with Dimension-five Operators $\left(M S S M_{5}\right)$," Nucl. Phys. B 808, 155 (2009) arXiv:0806.3778 [hep-ph]].

[13] A. Strumia, "Bounds on Kaluza-Klein excitations of the SM vector bosons from electroweak tests," Phys. Lett. B 466, 107 (1999) arXiv:hep-ph/9906266.

[14] J. A. Casas, J. R. Espinosa and I. Hidalgo, "The MSSM fine tuning problem: A Way out," JHEP 0401, 008 (2004) arXiv:hep-ph/0310137.

[15] S. Cassel, D. M. Ghilencea and G. G. Ross, "Fine tuning as an indication of physics beyond the MSSM," arXiv:0903.1115 [hep-ph].

[16] K. Blum and Y. Nir, "Beyond MSSM Baryogenesis," Phys. Rev. D 78, 035005 (2008) arXiv:0805.0097 [hep-ph]].

[17] P. Batra and E. Ponton, "Supersymmetric electroweak symmetry breaking," Phys. Rev. D 79, 035001 (2009) [arXiv:0809.3453 [hep-ph]].

[18] S. P. Martin, "Dimensionless supersymmetry breaking couplings, flat directions, and the origin of intermediate mass scales," Phys. Rev. D 61, 035004 (2000) arXiv:hep-ph/9907550.

[19] Z. Komargodski and N. Seiberg, "Comments on the Fayet-Iliopoulos Term in Field Theory and Supergravity," arXiv:0904.1159 [hep-th].

[20] J. A. Casas, J. R. Espinosa and I. Navarro, "Unconventional low-energy SUSY from warped geometry," Nucl. Phys. B 620, 195 (2002) arXiv:hep-ph/0109127.

[21] D. Piriz and J. Wudka, "Effective operators in supersymmetry," Phys. Rev. D 56, 4170 (1997) arXiv:hep-ph/9707314.

[22] S. R. Coleman, "The Fate Of The False Vacuum. 1. Semiclassical Theory," Phys. Rev. D 15, 2929 (1977) [Erratum-ibid. D 16, 1248 (1977)].

[23] S. R. Coleman, V. Glaser and A. Martin, "Action Minima Among Solutions To A Class Of Euclidean Scalar Field Equations," Commun. Math. Phys. 58, 211 (1978).

[24] A. H. Guth and E. J. Weinberg, "Cosmological Consequences Of A First Order Phase Transition In The SU(5) Grand Unified Model," Phys. Rev. D 23, 876 (1981).

[25] T. Banks and C. M. Bender, "Coupled anharmonic oscillators. ii. unequal-mass case," Phys. Rev. D 8, 3366 (1973).

[26] M. J. Duncan and L. G. Jensen, "Exact tunneling solutions in scalar field theory," Phys. Lett. B 291, 109 (1992).

[27] C. Amsler et al. [Particle Data Group], "Review of particle physics," Phys. Lett. B 667 (2008) 1. 